Internat. J. Math. \& Math. Sci.

Vol. 22, No. 3 (1999) 649-654

S 0161-1712〈99>22649-X

(c) Electronic Publishing House

\title{
SOME APPLICATIONS OF A DIFFERENTIAL SUBORDINATION
}

\author{
YONG CHAN KIM and H. M. SRIVASTAVA
}

(Received 14 January 1998)

\begin{abstract}
A number of interesting criteria were given by earlier workers for a normalized analytic function to be in the familiar class $\varphi^{*}$ of starlike functions. The main object of the present paper is to extend and improve each of these earlier results. An application associated with an integral operator $\mathscr{F}_{c}(c>-1)$ is also considered.
\end{abstract}

Keywords and phrases. Differential subordination, analytic functions, starlike functions, integral operator, Gauss hypergeometric function, Digamma function.

1991 Mathematics Subject Classification. Primary 30C45; Secondary 33B15, 33C05.

1. Introduction. Let $\mathscr{A}(n)$ denote the class of functions of the form

$$
f(z)=z+\sum_{k=n+1}^{\infty} a_{k} z^{k}, \quad(n \in \mathbb{N}:=\{1,2,3, \ldots\})
$$

which are analytic in the open unit disk $U=\{z: z \in \mathbb{C}$ and $|z|<1\}$. Also, let $\mathscr{S}^{*}$ be the class of starlike functions in $\mathcal{U}$, defined by (cf., e.g., $[2,11])$

$$
\mathscr{S}^{*}:=\left\{f(z) \in \mathscr{A}(1): \mathfrak{R}\left(\frac{z f^{\prime}(z)}{f(z)}\right)>0,(z \in \mathcal{U})\right\} .
$$

For analytic functions $g(z)$ and $h(z)$ with $g(0)=h(0), g(z)$ is said to be subordinate to $h(z)$ if there exists an analytic function $w(z)$ such that $w(0)=0,|w(z)|<1$, $(z \in \mathcal{U})$, and $g(z)=h(w(z))$. We denote this subordination by $g(z) \prec h(z)$.

For a function $f(z)$ belonging to the class $\mathscr{A}(1)$, Bernardi [1] defined the integral operator $\mathscr{F}_{C}$ as follows:

$$
\left(\mathscr{F}_{c} f\right)(z)=\frac{c+1}{z^{c}} \int_{0}^{z} t^{c-1} f(t) d t, \quad(c>-1 ; z \in \mathcal{U}) .
$$

We note that $\mathscr{F}_{c} f \in \mathscr{A}(n)$ if $f \in \mathscr{A}(n)$. In particular, the operator $\mathscr{F}_{1}$ was studied earlier by Libera [3]. (Also, see Owa and Srivastava [8, p. 126 et seq.]).

R. Singh and S. Singh [10] proved that if $f(z) \in \mathscr{A}(1)$ and

$$
\mathfrak{R}\left\{f^{\prime}(z)+z f^{\prime \prime}(z)\right\}>-\frac{1}{4}, \quad(z \in \mathcal{U}),
$$

then $f(z) \in \mathscr{S}^{*}$.

Recently, Yi and Ding [12] improved the above-mentioned result of R. Singh and S. Singh [10] by showing that if $f(z) \in \mathscr{A}(1)$ and

$$
\mathfrak{R}\left\{f^{\prime}(z)+z f^{\prime \prime}(z)\right\}>1-\frac{3}{4(1-\log 2)^{2}+2} \approx-0.263, \quad(z \in \mathcal{u}),
$$


then $f(z) \in \mathscr{S}^{*}$.

Furthermore, Nunokawa and Thomas [6] proved that if $f(z) \in \mathscr{A}(1)$ and

$$
\mathfrak{R}\left\{f^{\prime}(z)\right\}>-0.0175 \ldots, \quad(z \in \mathcal{U}),
$$

then $\mathscr{F}_{1} f \in \mathscr{S}^{*}$

In this paper, we extend and improve each of these earlier results in [6,12] and also consider an interesting application associated with the integral operator $\mathscr{F}_{c}$.

2. Preliminary results. The following results are required in our investigation.

LEMMA 1 (Yi and Ding [12, Lem. 1]). Suppose that the function $\phi: \mathbb{C}^{2} \times \mathfrak{U} \rightarrow \mathbb{C}$ satisfies the condition $\mathfrak{R}\{\phi(i x, y ; z)\} \leq \delta$ for all real $x$ and $y \leq-(1 / 2)\left(1+x^{2}\right)$ and all $z \in \mathcal{U}$. If $p(z)=1+p_{1} z+p_{2} z+\cdots$ is analytic in $u$ and

$$
\mathfrak{R}\left\{\phi\left(p(z), z p^{\prime}(z) ; z\right)\right\}>\delta, \quad(z \in \mathcal{U}),
$$

then $\mathfrak{R}\{p(z)\}>0$ in $U$.

LEMMA 2 (Owa and Nunokawa [7, Thm. 1]). Let $p(z)$ be analytic in $u$ with

$$
p(0)=1, \quad p^{\prime}(0)=\cdots=p^{(n-1)}(0)=0 .
$$

If $p(z)$ satisfies the inequality

$$
\mathfrak{R}\left\{p(z)+\alpha z p^{\prime}(z)\right\}>\beta, \quad(z \in \mathcal{U}),
$$

then

$$
\mathfrak{R}\{p(z)\}>\beta+(1-\beta)\left\{2 \int_{0}^{1} \frac{d \rho}{1+\rho^{n \mathfrak{R}(\alpha)}}-1\right\}, \quad(z \in \mathcal{U}),
$$

where $\alpha \neq 0, \mathfrak{R}(\alpha) \geq 0$, and $\beta<1$.

LEMMA 3 (Owa and Nunokawa [7, Ex. 1]). Let $\alpha>0$ and $\beta<1$. If $f(z) \in \mathscr{A}(n)$ satisfies the inequality

$$
\mathfrak{R}\left\{f^{\prime}(z)+\alpha z f^{\prime \prime}(z)\right\}>\beta, \quad(z \in \mathcal{U}),
$$

then

$$
\mathfrak{R}\left\{f^{\prime}(z)\right\}>\beta+(1-\beta)\{2 \delta(n, \alpha)-1\}, \quad(z \in \mathcal{U}),
$$

where

$$
\delta(n, \alpha)=\int_{0}^{1} \frac{d \rho}{1+\rho^{n \alpha}} .
$$

Incidentally, the value of $\delta(n, \alpha)$ in (2.7) can be expressed as the Gauss hypergeometric function

$$
{ }_{2} F_{1}\left(1, \frac{1}{n \alpha} ; 1+\frac{1}{n \alpha} ;-1\right)
$$

which may also be rewritten in terms of the difference of two Digamma (or $\psi$-) functions

$$
\frac{1}{2 n \alpha}\left[\psi\left(\frac{1+n \alpha}{2 n \alpha}\right)-\psi\left(\frac{1}{2 n \alpha}\right)\right] \quad\left(\psi(z):=\frac{\Gamma^{\prime}(z)}{\Gamma(z)}\right) .
$$

We also note that the inequality (2.5) is equivalent to the subordination given by 


$$
f^{\prime}(z)+\alpha z f^{\prime \prime}(z) \prec \frac{1+(1-2 \beta) z}{1-z} .
$$

3. Main results. The following theorem is a generalization of the main result of Yi and Ding [12].

Theorem. Let $\delta(n, \alpha)$ be as defined in Lemma 3 and let $\theta=0.911621907, \alpha \geq$ 0.17418 , and

$$
\alpha-\frac{(1-\alpha)^{2}}{3 \alpha} \tan ^{2} \theta<\frac{2 \delta(n, \alpha)-1}{\{1-\delta(n, \alpha)\}\{2 \delta(n, 1)-1\}} .
$$

If $f \in \mathscr{A}(n)$ satisfies the inequality

$$
\begin{aligned}
& \mathfrak{R}\left\{f^{\prime}(z)+\alpha z f^{\prime \prime}(z)\right\} \\
& >1-\frac{\frac{2}{\alpha}+\left(1-\frac{(1-\alpha)^{2}}{3 \alpha^{2}} \tan ^{2} \theta\right)}{\frac{2}{\alpha}+4\{1-\delta(n, 1)\}\{1-\delta(n, \alpha)\}\left(1-\frac{(1-\alpha)^{2}}{3 \alpha^{2}} \tan ^{2} \theta\right)}, \quad(z \in U),
\end{aligned}
$$

then $f(z) \in \mathscr{S}^{*}$.

Proof. Making use of Lemma 3 and the inequality (3.2), we obtain

$$
\begin{aligned}
\mathfrak{R}\left\{f^{\prime}(z)\right\} & >\beta+(1-\beta)\{2 \delta(n, \alpha)-1\} \\
& =2\{\delta(n, \alpha)-1\}\left[\frac{\frac{2}{\alpha}+\left(1-\frac{(1-\alpha)^{2}}{3 \alpha^{2}} \tan ^{2} \theta\right)}{\frac{2}{\alpha}+4\{1-\delta(n, 1)\}\{1-\delta(n, \alpha)\}\left(1-\frac{(1-\alpha)^{2}}{3 \alpha^{2}} \tan ^{2} \theta\right)}\right]+1 \\
& =: \gamma, \quad(z \in U),
\end{aligned}
$$

where

$$
\beta=1-\frac{\frac{2}{\alpha}+\left(1-\frac{(1-\alpha)^{2}}{3 \alpha^{2}} \tan ^{2} \theta\right)}{\frac{2}{\alpha}+4\{1-\delta(n, 1)\}\{1-\delta(n, \alpha)\}\left(1-\frac{(1-\alpha)^{2}}{3 \alpha^{2}} \tan ^{2} \theta\right)} .
$$

Since $\alpha \geq 0.17418$ and

$$
\frac{1}{2}<\delta(n, \alpha)<1, \quad(\alpha>0 ; n \in \mathbb{N}),
$$

we have

$$
\frac{2}{\alpha}+4\{1-\delta(n, 1)\}\{1-\delta(n, \alpha)\}\left(1-\frac{(1-\alpha)^{2}}{3 \alpha^{2}} \tan ^{2} \theta\right)>0 .
$$

Hence, by (3.1), we find from (3.3) that

$$
0<\gamma<1 .
$$

If we put $p(z)=z^{-1} f(z)$, then

$$
\mathfrak{R}\left\{f^{\prime}(z)\right\}=\mathfrak{R}\left\{p(z)+z p^{\prime}(z)\right\}>\gamma, \quad(z \in \mathcal{U}),
$$


which, in view of Lemma 2, implies that

$$
\mathfrak{R}\left\{\frac{f(z)}{z}\right\}>\gamma+(1-\gamma)\{2 \delta(n, 1)-1\}, \quad(z \in \mathcal{u}) .
$$

By using (3.5) and (3.7), we get

$$
\mathfrak{R}\left\{\frac{f(z)}{z}\right\}>0, \quad(z \in \mathcal{U}) .
$$

Next, we let

$$
q(z)=\frac{z f^{\prime}(z)}{f(z)} \quad \text { and } \quad \lambda(z)=\frac{f(z)}{z} .
$$

Then

$$
\mathfrak{R}\{\lambda(z)\}>\gamma+(1-\gamma)\{2 \delta(n, 1)-1\}, \quad(z \in \mathcal{U})
$$

and

$$
\begin{aligned}
f^{\prime}(z)+\alpha z f^{\prime \prime}(z) & =\lambda(z)\left[\alpha z q^{\prime}(z)+(1-\alpha) q(z)+\alpha\{q(z)\}^{2}\right] \\
& =\phi\left(q(z), z q^{\prime}(z) ; z\right)
\end{aligned}
$$

where $\phi(u, v ; z)=\lambda(z)\left[\alpha u^{2}+(1-\alpha) u+\alpha v\right]$.

By setting $\lambda(z)=a+b i$, we get

$$
\begin{aligned}
\mathfrak{R}\{\phi(i x, y ; z)\} & \leq-\frac{1}{2}\left\{3 \alpha a x^{2}+2 b(1-\alpha) x+\alpha a\right\} \\
& \leq-\frac{a}{2}\left\{\alpha-\frac{1}{3 \alpha}(1-\alpha)^{2}\left(\frac{b}{a}\right)^{2}\right\}
\end{aligned}
$$

for all real $x$ and $y \leq-(1 / 2)\left(1+x^{2}\right)$. Since $\mathfrak{R}\left\{f^{\prime}(z)\right\}>0(z \in \mathcal{U})$ implies that $\lambda(z) \prec L(z):=-1-(2 / z) \log (1-z)$, we have $\lambda(\mathcal{U}) \subset L(\mathcal{U})$, where (see [9])

$L(U) \subset\{\omega: \mathfrak{R}(\omega)>2 \log 2-1\} \cap\{\omega:|\mathfrak{I}(\omega)|<\pi\} \cap\{\omega:|\arg (\omega)|<\theta=0.911621907\}$.

By using (3.9) and (3.14), we obtain

$$
\begin{aligned}
\mathfrak{R}\{\phi(i x, y ; z)\} & \leq-\frac{a}{2}\left\{\alpha-\frac{(1-\alpha)^{2}}{3 \alpha} \tan ^{2} \theta\right\} \\
& \leq \beta, \quad(z \in \mathcal{U}) .
\end{aligned}
$$

Hence, by Lemma 1, we get

$$
\mathfrak{R}\{q(z)\}=\mathfrak{R}\left\{\frac{z f^{\prime}(z)}{f(z)}\right\}>0, \quad(z \in U) .
$$

This evidently completes the proof of the theorem.

COROLLARY 1. Let $\theta=0.911621907, \alpha \geq 0.17418$, and

$$
\alpha-\frac{(1-\alpha)^{2}}{3 \alpha} \tan ^{2} \theta<\frac{2 \delta(1, \alpha)-1}{\{1-\delta(1, \alpha)\}(2 \log 2-1)} .
$$


If $f \in \mathscr{A}(1)$ satisfies the inequality

$$
\begin{aligned}
& \mathfrak{R}\left\{f^{\prime}(z)+\alpha z f^{\prime \prime}(z)\right\} \\
& >1-\frac{\frac{2}{\alpha}+\left(1-\frac{(1-\alpha)^{2}}{3 \alpha^{2}} \tan ^{2} \theta\right)}{\frac{2}{\alpha}+4(1-\log 2)\{1-\delta(1, \alpha)\}\left(1-\frac{(1-\alpha)^{2}}{3 \alpha^{2}} \tan ^{2} \theta\right)}, \quad(z \in \mathcal{U}),
\end{aligned}
$$

then $f(z) \in \mathscr{S}^{*}$.

REMARK 1. For $\alpha=1$, Corollary 1 immediately yields the main result of Yi and Ding [12, Thm., p. 614].

REMARK 2. A result of Ponnusamy [9, Thm. 4] can be obtained by taking $\beta=0$ in the proof of our theorem.

It is not difficult to apply the definition (1.3) in order to show that

$$
f^{\prime}(z)=\left(\mathscr{F}_{C} f\right)^{\prime}(z)+\frac{1}{c+1} z\left(\mathscr{F}_{C} f\right)^{\prime \prime}(z) .
$$

Thus, by the theorem, we arrive at the following application:

Corollary 2. Let $\theta=0.911621907,-1<c \leq 4.741187$, and

$$
\frac{1}{c+1}-\frac{c^{2}}{3(c+1)} \tan ^{2} \theta<\frac{2 \delta\left(n, \frac{1}{c+1}\right)-1}{\left\{1-\delta\left(1, \frac{1}{c+1}\right)\right\}\{2 \delta(n, 1)-1\}} .
$$

If $f \in \mathscr{A}(n)$ satisfies the inequality

$$
\mathfrak{R}\left\{f^{\prime}(z)\right\}>1-\frac{2(c+1)+\left(1-\frac{1}{3} c^{2} \tan ^{2} \theta\right)}{2(c+1)+4\{1-\delta(n, 1)\}\left\{1-\delta\left(n, \frac{1}{c+1}\right)\right\}\left(1-\frac{1}{3} c^{2} \tan ^{2} \theta\right)}, \quad(z \in u)
$$

then $\mathscr{F}_{c} f \in \mathscr{S}^{*}$, where $\mathscr{F}_{c}$ is defined by (1.3).

By setting $c=n=1$ in Corollary 2, we obtain Corollary 3 below, which shows that the constant -0.0175 in the inequality (1.6) of Nunokawa and Thomas [6] can be reduced further.

COROLLARY 3. Let $\theta=0.911621907$. If $f \in \mathscr{A}(1)$ satisfies the inequality

$$
\mathfrak{R}\left\{f^{\prime}(z)\right\}>1-\frac{5-(1 / 3) \tan ^{2} \theta}{4+8(1-\log 2)^{2}\left(1-(1 / 3) \tan ^{2} \theta\right)} \approx-0.025311 \ldots, \quad(z \in \mathcal{U}),
$$

then $\mathscr{F}_{1} f \in \mathscr{S}^{*}$.

Proof. Since

$\frac{1}{2}-\frac{1}{6} \tan ^{2} \theta=0.222356(\theta=0.911621907) \quad$ and $\quad \frac{3-4 \log 2}{(2 \log 2-1)^{2}}=1.523967 \ldots$,

the proof of Corollary 3 is completed by setting $c=n=1$ in Corollary 2 . 
REMARK 3. Several nonsharp results, obtained by various other authors (cf., e.g., [9]), correspond to the further special cases of Corollary 2 when $c=0$ and $c=1$.

ACKNOWLEDGEMENTS. The present investigation was initiated during the secondnamed author's visit to Yeungnam University in December 1996. This work was partially supported by KOSEF and BSRI-97-1401 of Korea and by the Natural Sciences and Engineering Research Council of Canada under Grant OGP 0007353.

\section{REFERENCES}

[1] S. D. Bernardi, Convex and starlike univalent functions, Trans. Amer. Math. Soc. 135 (1969), 429-446. MR 38\#1243. Zbl 172.09703.

[2] P. L. Duren, Univalent Functions, Grundlehren der Mathematischen Wissenschaften, vol. 259, Springer-Verlag, New York, Berlin, 1983. MR 85j:30034. Zbl 514.30001.

[3] R. J. Libera, Some classes of regular univalent functions, Proc. Amer. Math. Soc. 16 (1965), 755-758. MR 31\#2389. Zbl 158.07702.

[4] S. Miller and P. T. Mocanu, Second-order differential inequalities in the complex plane, J. Math. Anal. Appl. 65 (1978), no. 2, 289-305. MR 80f:30009. Zbl 379.34003.

[5] M. Nunokawa, On starlikeness of Libera transformation, Complex Variables Theory Appl. 17 (1991), no. 1-2, 79-83. MR 92k:30021. Zbl 758.30011.

[6] M. Nunokawa and D. K. Thomas, On the Bernardi integral operator, Current topics in analytic function theory (H. M. Srivastava and S. Owa, Editors) (River Edge, N.J., Singapore, London, Hong Kong), World Sci. Publishing, 1992, pp. 212-219. MR 94m:30028.

[7] S. Owa and M. Nunokawa, Applications of a subordination theorem, J. Math. Anal. Appl. 188 (1994), no. 1, 219-226. MR 95i:30022. Zbl 817.30009.

[8] S. Owa and H. M. Srivastava, Some applications of the generalized Libera integral operator, Proc. Japan Acad. Ser. A Math. Sci. 62 (1986), no. 4, 125-128. MR 88a:30027. Zbl 583.30016.

[9] S. Ponnusamy, Differential subordination and starlike functions, Complex Variables Theory Appl. 19 (1992), no. 3, 185-194. CMP 94 15. Zbl 756.30018.

[10] R. Singh and S. Singh, Convolution properties of a class of starlike functions, Proc. Amer. Math. Soc. 106 (1989), no. 1, 145-152. MR 90d:30037. Zbl 672.30007.

[11] H. M. Srivastava and S. Owa (eds.), Current Topics in Analytic Function Theory, World Scientific Publishing Co., Inc., Singapore, River Edge, N.J., 1992. MR 94b:30001. Zbl 970.22308.

[12] L. Yi and S. Ding, A new criterion for starlike functions, Internat. J. Math. Math. Sci. 19 (1996), no. 3, 613-614. MR 97b:30019. Zbl 849.30007.

Kim: Department of MATHematics, College of Education, Yeungnam University, 214-1

DAEDONG, GYONGSAN 712-749, KOREA

E-mail address: kimyc@ynucc.yeungnam.ac.kr

SRivastaVA: Department of Mathematics and Statistics, University of Victoria, VICTORIA, BRITISH COLUMBIA V8W 3P4, CANADA

E-mail address: hmsri@uvvm.uvic.ca 


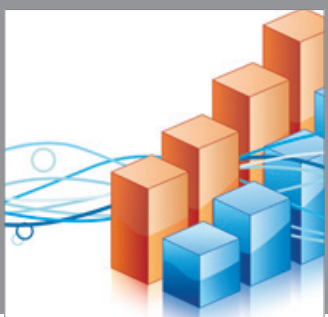

Advances in

Operations Research

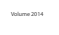

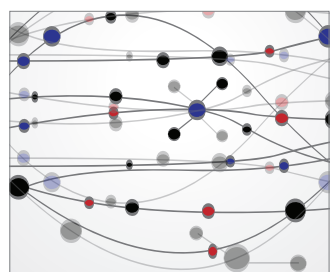

\section{The Scientific} World Journal
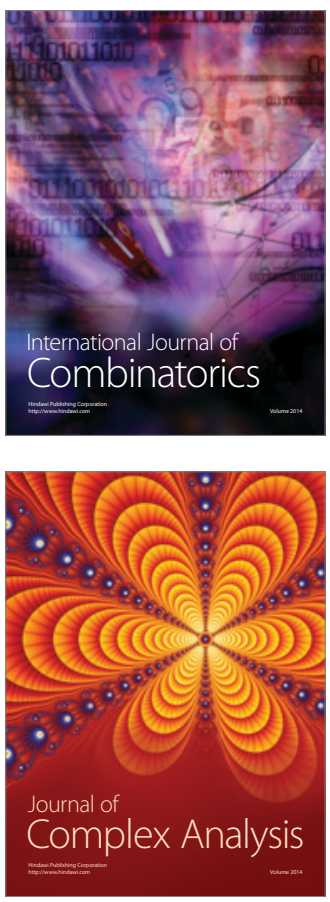

International Journal of

Mathematics and

Mathematical

Sciences
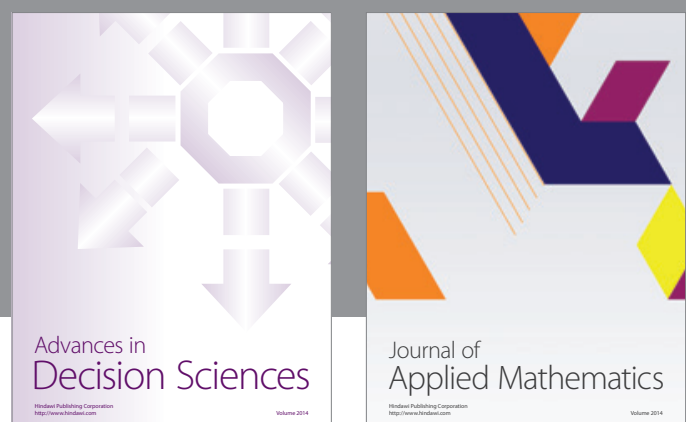

Journal of

Applied Mathematics
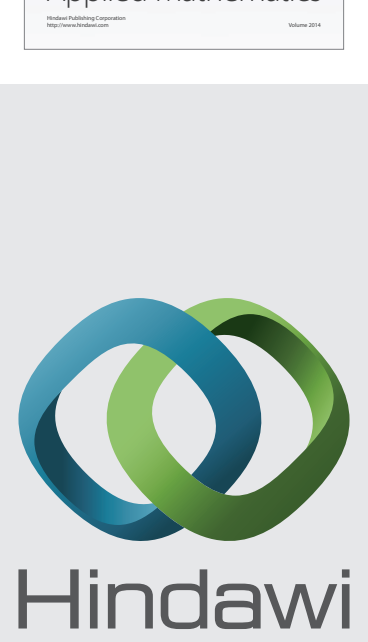

Submit your manuscripts at http://www.hindawi.com
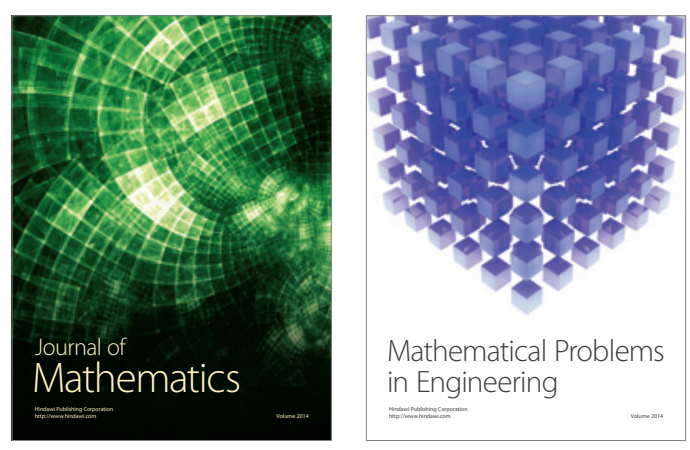

Mathematical Problems in Engineering
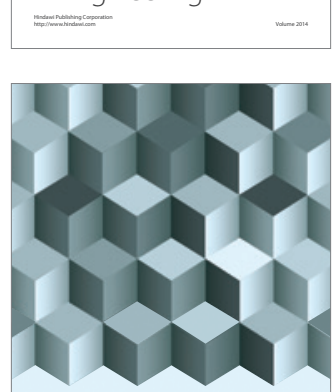

Journal of

Function Spaces
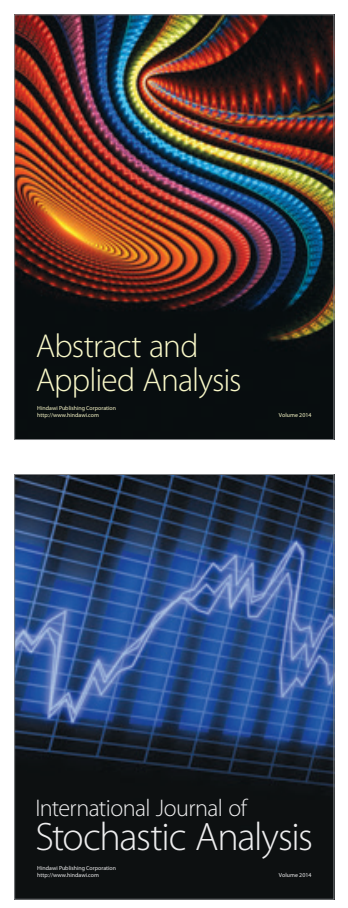

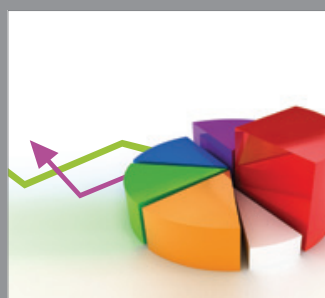

ournal of

Probability and Statistics

Promensencen
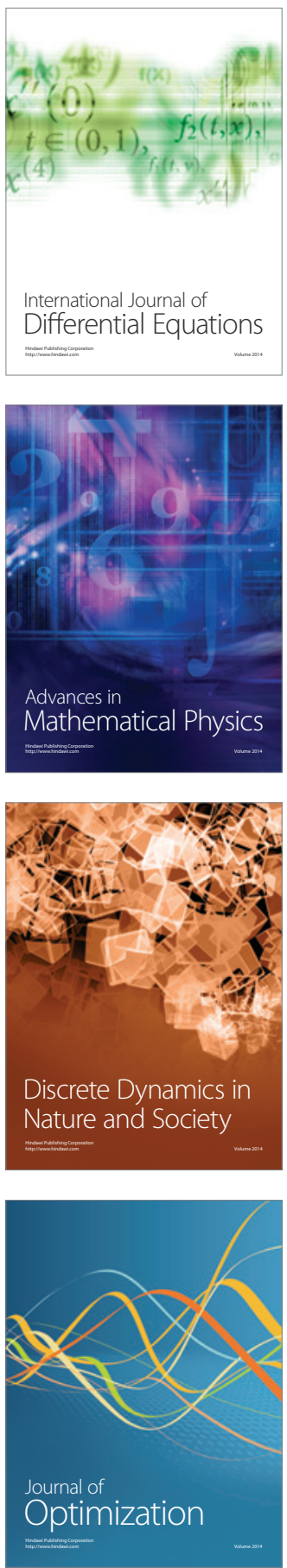